TITLE:

\title{
The Maximum Latency and Identification of Positive Boolean Functions
}

\author{
$\operatorname{AUTHOR}(\mathrm{S})$ :
}

MAKINO, Kazuhisa; IBARAKI, Toshihide

\section{CITATION:}

MAKINO, Kazuhisa ... [et al]. The Maximum Latency and Identification of Positive Boolean Functions. 数理解析研究所講究録 1994, 871: 73-79

ISSUE DATE:

1994-05

URL:

http://hdl.handle.net/2433/84054

RIGHT: 


\title{
The Maximum Latency and Identification of Positive Boolean Functions 正論理関数の最大潜伏度と同定

\author{
Kazuhisa MAKINO and Toshihide IBARAKI
} 牧野和久 茨木俊秀
}

Department of Applied Mathematics and Physics, Faculty of Engineering, Kyoto University

\begin{abstract}
Consider the problem of identifying $\min T(f)$ and $\max F(f)$ of a positive (i.e., monotone) Boolean function $f$, by using membership queries only, where $\min T(f)(\max F(f))$ denotes the set of minimal true vectors (maximal false vectors) of $f$. It is known that an incrementally polynomial algorithm exists if and only if there is a polynomial time algorithm to check the existence of an unknown vector for given sets $M T \subseteq \min T(f)$ and $M F \subseteq \max F(f)$. Unfortunately, however, the complexity of this problem is still unknown. To answer this question partially, we introduce in this paper a measure for the difficulty of finding an unknown vector, which is called the maximum latency. If the maximum latency is constant, then an unknown vector can be found in polynomial time and there is an incrementally polynomial algorithm for identification. Several subclasses of positive functions are shown to have constant maximum latency, e.g., 2-monotonic positive functions, $\Delta$-partial positive threshold functions and matroid functions, while the class of general positive functions has maximum latency not smaller than $\lfloor n / 4\rfloor+1$ and the class of positive $k$-DNF functions has $\Omega(\sqrt{n})$ maximum latency.
\end{abstract}

\section{Introduction}

Consider the problem of identifying $T(f)$ (set of true vectors) and $F(f)$ (set of false vectors) of a given Boolean function (or a function in short) $f$ by asking membership queries to an oracle whether $f(u)=0$ or 1 holds for some selected vectors $u$ [2]. In the terminology of computational learning theory $[1,12]$, this is the exact learning of a Boolean theory $f$ by membership queries only. It is also a process of forming a theory that explains a certain phenomenon by collecting positive and negative data (in the sense of causing and not causing that phenomenon) [4]. In particular, we are interested in the case where $f$ is known to be positive, i.e., monotone. If $f$ is a positive function, $T(f)$ and $F(f)$ can be compactly represented by $\min T(f)$ (set of minimal true vectors) and $\max F(f)$ (set of maximal false vectors). Therefore our problem is stated as follows.
Input: an oracle for a positive function $f$.

Output: $\min T(f)$ and $\max F(f)$.

The complexity of this type of enumeration algorithm is usually measured in its length of input and output. An algorithm to enumerate items $a_{1}, a_{2}, \ldots, a_{p}$ is called incrementally polynomial [7], (i) if it iterates the following procedure for $i=1,2, \ldots, p$ : output the $i$-th item $a_{i}$ from the knowledge of its input and items $a_{1}, a_{2}, \ldots, a_{i-1}$ generated by then, and (ii) if the time required for the $i$-th iteration is polynomial in the input length and the sizes of $a_{1}, a_{2}, \ldots, a_{i-1}$. If an algorithm is incrementally polynomial, it also satisfies the criterion of polynomial total time [7] (i.e., polynomial time in the length of input and output).

Now let $M T$ and $M F$ respectively denote the partial knowledge of $\min T(f)$ and $\max F(f)$ currently at hand, i.e.,

$M T \subseteq \min T(f)$ and $M F \subseteq \max F(f)$. 
Define

$$
\begin{aligned}
& T(M T)=\{v \mid v \geq w \text { for some } w \in M T\} \\
& F(M F)=\{v \mid v \leq w \text { for some } w \in M F\} .
\end{aligned}
$$

By assumption (1.1), $T(M T) \subseteq T(f)$ and $F(M F) \subseteq F(f)$, and hence

$$
T(M T) \cap F(M F)=\emptyset
$$

holds. A vector $u$ is called unknown if

$$
u \in\{0,1\}^{n} \backslash(T(M T) \cup F(M F)),
$$

since it is not known at the current stage whether $u$ is a true vector or a false vector of $f$. If there is no unknown vector, then $T(M T) \cup F(M F)=$ $\{0,1\}^{n}$ holds, i.e., $M T=\min T(f)$ and $M F=$ $\max F(f)$ hold for some positive function $f$.

The general procedure of identifying a positive function $f$ can be described as follows.

\section{Algorithm IDENTIFY}

Input: an oracle for a positive function $f$.

Output: $\min T(f)$ and $\max F(f)$.

1. Start with appropriate sets $M T(\subseteq \min$ $T(f))$ and $M F(\subseteq \max F(f))$, where $M T \cup$ $M F \neq \phi$ is assumed.

2. Test if $T(M T) \cup F(M F)=\{0,1\}^{n}$ holds. If so, output $M T$ and $M F$, and halt. Otherwise, find an unknown vector $u$ and go to 3 .

3. Ask an oracle if $f(u)=1$ or $f(u)=0$. If $f(u)=1$, then compute a new minimal true vector $y$ such that $y \leq u$ and let $M T:=M T \cup$ $\{y\}$. On the other hand, if $f(u)=0$, compute a new maximal false vector $y$ such that $y \geq u$ and let $M F:=M F \cup\{y\}$. Return to 2 .

The crucial part of this algorithm is in Step 2, i.e., to solve the following problem, where a set of vectors $M$ is incomparable if any pair of vectors $v$ and $w$ in $M$ satisfies $v \nsupseteq w$ and $w \nsupseteq v$.

\section{Problem EQ}

Input: Incomparable sets $M T, M F\left(\subseteq\{0,1\}^{n}\right)$ such that $T(M T) \cap F(M F)=\emptyset$.

Question: Does $T(M T) \cup F(M F)=\{0,1\}^{n}$ (i.e., no unknown vector) hold?

If problem EQ can be solved in polynomial time, it is known that an unknown vector in
Step 2 can be found in polynomial time [2], and that computing a minimal true vector or a maximal false vector $y$ from an unknown vector $u$ in Step 3 can also be done in polynomial time $[1,2,12]$. Therefore, an incrementally polynomial algorithm exists if and only if problem EQ can be solved in polynomial time. It is shown in [2] that problem EQ is polynomially equivalent to many other interesting problems encountered in various fields such as hypergraph theory [5], theory of coteries (used in distributed systems) [6], artificial intelligence [11] and Boolean theory [2]. Unfortunately, the complexity of these problems is still open, though there is some evidence to conjecture that it is co NP-complete.

In order to investigate the complexity of $\mathrm{EQ}$ for subclasses of positive functions, we introduce in this paper the concept of maximum latency, which is a complexity measure for finding an unknown vector. If the maximum latency is constant, then it is shown in section 2 that EQ can be solved in polynomial time, though the converse is not generally true. In section 4 , we show that the maximum latency of general positive functions is at least $\lfloor n / 4\rfloor+1$. However, several special classes of positive functions are found in section 3 to have constant maximum latency; classes of (i) 2-monotonic positive functions $[3,10]$, (ii) $\Delta$ partial positive threshold functions [10], (iii) matroid functions [13], (iv) $k$-tight functions, and (v) others. For these classes of positive functions, therefore, there are incrementally polynomial identification algorithms. Finally it is shown in section 4 that the class of positive $k$-DNF functions has the maximum latency of $\Omega(\sqrt{n})$, even though it is known [5] that EQ can be solved in polynomial time for this class of functions.

The last result indicates that the concept of maximum latency is not always sufficient to distinguish polynomially solvable cases from those not solvable in polynomial time. However, it is also evident that the maximum latency is a powerful tool to find polynomially solvable special cases. 


\section{Definitions and basic proper- ties}

A Boolean function, or a function in short, is a mapping $f:\{0,1\}^{n} \mapsto\{0,1\}$, where $v \in\{0,1\}^{n}$ is called a Boolean vector (a vector in short). If $f(v)=1$ (resp. 0), then $v$ is called a true (resp. false) vector of $f$. The set of all true vectors (false vectors) is denoted by $T(f)(F(f))$. A function $f$ is positive if $v \leq w$ always implies $f(v) \leq f(w)$. A positive function is also called monotone. A true vector $v$ of $f$ is minimal if there is no other true vector $w$ such that $w<v$, and let $\min T(f)$ denote the set of all minimal true vectors of $f$. A maximal false vector is symmetrically defined and $\max F(f)$ denotes the set of all maximal false vectors of $f$.

If $f$ is positive, it is known that $f$ has the unique disjunctive normal form (DNF) consisting of all prime implicants. There is one-to-one correspondence between prime implicants and minimal true vectors. For example, a positive function $f=x_{1} x_{2} \vee x_{2} x_{3} \vee x_{3} x_{1}$, has prime implicants $x_{1} x_{2}, x_{2} x_{3}, x_{3} x_{1}$ which correspond to minimal true vectors (110), (011), (101), respectively. The input length to describe a positive function $f$ is $O(n|\min T(f)|)$ if it is represented in this manner.

Given incomparable sets $M T, M F\left(\subseteq\{0,1\}^{n}\right)$ such that $M T \cup M F \neq \emptyset$ and $T(M T) \cap F(M F)=$ $\emptyset$, the partial function $g$ is defined by

$$
g(v)= \begin{cases}1, & v \in T(M T) \\ 0, & v \in F(M F) \\ \text { unknown, } & \text { otherwise. }\end{cases}
$$

If $M T$ and $M F$ of $g$ satisfy $M T \subseteq \min T(f)$ and $M F \subseteq \max F(f)$ for some (complete) positive function $f$, then $g$ is called a partial function of $f$. The set of unknown vectors of $g$ is denoted by $U(g)$, i.e.,

$$
U^{\prime}(g)=\{0,1\}^{n} \backslash(T(M T) \cup F(M F)) .
$$

The $k$-neighborhood of $g$ is defined by

$$
\begin{aligned}
& N_{k}(g)=\{v \mid\|v-a\| \leq k \\
& \quad \text { for some } a \in M T \cup M F\},
\end{aligned}
$$

where $\|w\|$ denotes $\sum_{i=1}^{n}\left|w_{i}\right|$. The latency of $g$, $\lambda(g)$, is defined to be the integer $k$ satisfying

$$
N_{k-1}(g) \cap U(g)=\phi \text { and } N_{k}(g) \cap U(g) \neq \phi .
$$

As a special case, if $U(g)=\phi$ i.e., $g=f$, then $\lambda(g)$ is defined to be $0 . \lambda(g)$ is equivalently given by

$$
\lambda(g)=\min \{\|u-a\| \mid a \in M T \cup M F, u \in U(g)\} .
$$

Now let $C_{X}$ be a subclass of positive functions. $C_{X}(n)$ denotes the set of functions in $C_{X}$ with $n$ variables. For $C_{X}(n)$, the maximum latency is defined by

$$
\begin{aligned}
& \Lambda_{X}(n)=\max \{\lambda(g) \mid g \text { is } \\
& \left.\quad \text { a partial function of } f \in C_{X}(n)\right\} .
\end{aligned}
$$

If $g$ is a partial function of $f \in C_{X}(n)$, then by definition there is no unknown vector if $N_{\Lambda_{X}(n)}(g) \cap U(g)=\phi$. That is, in order to find an unknown vector, we only need to search $\Lambda_{X}(n)$-neighborhood of $g$. Therefore, if a positive function $f$ of $n$ variables is known to belong to class $C_{X}(n)$, Step 2 of Algorithm IDENTIFY can be executed as follows.

2. Test if $N_{\Lambda_{X}(n)}(g) \subseteq T(M T) \cup F(M F)$, where $g$ is the partial function defined by $M T$ and $M F$. If so, output $M T$ and $M F$, and halt. Otherwise, find an unknown vector $u \in N_{\Lambda_{X}(n)}(g) \backslash(T(M T) \cup F(M F))$ and go to 3 .

The test of $N_{\Lambda_{X}(n)}(g) \subseteq T(M T) \cup F(M F)$ can be accomplished by checking if $v \geq a$ for some $a \in M T$ or $v \leq b$ for some $b \in M F$, for every $v \in N_{\Lambda_{X(n)}}(g)$. This computation takes at most

$$
\begin{aligned}
n(|M T|+|M F|) \mid & N_{\Lambda_{X}(n)}(g) \mid= \\
& n(|M T|+|M F|)^{2} n^{\Lambda_{X}(n)}
\end{aligned}
$$

time. Therefore, we have the next result.

Theorem 2.1 Let $g$, defined by $M T$ and $M F$, be a partial function of $f \in C_{X}(n)$. If $\Lambda_{X}(n)$ is constant, then the above Step 2 can be executed in polynomial time in $n$ and $|M T|+|M F|$. ( Therefore, problem EQ can be solved in polynomial time, and there is an incrementally polynomial algorithm to identify $f \in C_{X}(n)$. ) 


\section{Restricted classes of positive functions with constant max- imum latencies}

In this section, we show that there are some nontrivial special classes of positive functions, which have constant maximum latency. These classes are important in practice and theory (e.g., $[5,10,13])$.

\subsection{2-monotonic positive functions and $\Delta$-partial positive threshold func- tions}

If functions $f$ and $g$ satisfy $g(a) \leq f(a)$ for any $a \in\{0,1\}^{n}$, then we denote $g \leq f$. If $g \leq f$ and there exists a vector $a$ satisfying $g(a)=1$ and $f(a)=0$, we denote $g<f$. An assignment $A$ of binary values 0 or 1 to $k$ variables $x_{i_{1}}, x_{i_{2}}, \ldots, x_{i_{k}}$ is called a $k$-assignment, and is denoted by

$$
A=\left(x_{i_{1}} \leftarrow a_{1}, x_{i_{2}} \leftarrow a_{2}, \ldots, x_{i_{k}} \leftarrow a_{k}\right),
$$

where each of $a_{1}, a_{2}, \ldots a_{k}$ is either 1 or 0 . Let the complement of $A$, denoted by $\bar{A}$, represent the assignment obtained from $A$ by complementing all the 1's and 0's in $A$. When a function $f$ of $n$ variables and a $k$-assignment $A$ are given,

$$
f_{A}=f_{\left(x_{i_{1}} \leftarrow a_{1}, x_{i_{2}} \leftarrow a_{2}, \ldots, x_{i_{k}} \leftarrow a_{k}\right)}
$$

denotes the function of $(n-k)$ variables obtained by fixing variables $x_{i_{1}}, x_{i_{2}}, \ldots, x_{i_{k}}$ as specified by A.

Let $f$ be a function of $n$ variables. If either $f_{A} \leq f_{\bar{A}}$ or $f_{A} \geq f_{\bar{A}}$ holds for every $k$-assignment $A$, then $f$ is said to be $k$-comparable. If $f$ is $k$ comparable for every $k$ such that $1 \leq k \leq m$, then $f$ is said to be $m$-monotonic. (For more detailed discussion on these topics, see [10] for example.) In particular, $f$ is 1 -monotonic if $f_{\left(x_{i} \leftarrow 1\right)} \geq f_{\left(x_{i} \leftarrow 0\right)}$ or $f_{\left(x_{i} \leftarrow 1\right)} \leq f_{\left(x_{i} \leftarrow 0\right)}$ holds for any $i \in\{1,2, \ldots, n\}$. A function $f$ is positive if and only if $f$ is 1 -monotonic and $f_{\left(x_{i} \leftarrow 1\right)} \geq$ $f_{\left(x_{i} \leftarrow 0\right)}$ holds for all $i$.

Now consider a 2-assignment $A=\left(x_{i} \leftarrow\right.$ $\left.1, x_{j} \leftarrow 0\right)$. If

$$
f_{A} \geq f_{\bar{A}}\left(\text { resp. } f_{A}>f_{\bar{A}}\right)
$$

holds, this is denoted $x_{i} \succeq_{f} x_{j}$ (resp. $x_{i} \succ_{f} x_{j}$ ). Variables $x_{i}$ and $x_{j}$ are said to be comparable if either $x_{i} \succeq_{f} x_{j}$ or $x_{i} \preceq_{f} x_{j}$ holds. When $x_{i} \succeq_{f} x_{j}$ and $x_{i} \preceq_{f} x_{j}$ hold simultaneously, it is denoted as $x_{i} \approx_{f} x_{j}$. If $f$ is 2 -monotonic, this binary relation $\succeq_{f}$ over the set of variables is known to be a total preorder.[10]. A 2-monotonic positive function $f$ of $n$ variables is called regular if

$$
x_{1} \succeq_{f} x_{2} \succeq_{f} \cdots \succeq_{f} x_{n} .
$$

Any 2-monotonic positive function becomes regular by permuting variables. Let

$$
\begin{aligned}
& C_{P}: \text { class of all positive functions, } \\
& C_{2 M}: \text { class of } 2 \text {-monotonic positive functions. }
\end{aligned}
$$

Theorem 3.1 Class $C_{2 M}$ satisfies

$$
\Lambda_{2 M}(n)=1 \text {. }
$$

Proof. Assume that a 2-monotonic positive function $f$ is regular without loss of generality, and that $g$ is a partial function of $f$ defined by $M T$ and $M F$. Assume that $N_{1}(g) \cap U(g)=\phi$ and $U(g) \neq \phi$. Take a $u \in \max U(g)$, where $\max U(g)$ is the set of maximal unknown vectors (i.e., $u+e_{j} \in T(M T)$ for all $\left.j \in O F F(u)\right)$. Let $j=\max \{i \mid i \in O F F(u)\}$. There exists $a \in$ $M T$ such that $a \leq u+e_{j}$. Then $a-e_{j} \in F(M F)$ by assumption $N_{1}(g) \cap U(g)=\phi$. Therefore, there exists $b \in M F$ such that $b \geq a-e_{j}$. For any $l \in O F F(u) \backslash\{j\}$,

$$
a-e_{j}+e_{l} \in T(f) \subseteq T(M T) \cup U(g)
$$

by regularity of $f$, and hence $b \geq a-e_{j}+e_{l}$, i.e., $b \leq u$. (i) If $b=u$, then $u \in F(M F)$ which is a contradiction. (ii) If $b<u$, then $u \in T(M T)$ by $N_{1}(g) \cap U(g)=\phi$, which is also a contradiction.

The 2-monotonicity was originally introduced in conjunction with threshold functions (e.g., [10]), where a positive function $f$ is threshold if there exist $n+1$ nonnegative real numbers $w_{1}, w_{2}, \ldots, w_{n}$ and $t$ such that:

$$
f(x)= \begin{cases}1, & \text { if } \sum w_{i} x_{i} \geq t \\ 0, & \text { if } \sum w_{i} x_{i}<t .\end{cases}
$$


As $w_{i} \geq w_{j}$ implies $x_{i} \succeq_{f} x_{j}$ and $w_{i}=w_{j}$ implies $x_{i} \approx_{f} x_{j}$, a threshold function is always 2-monotonic, although the converse is not true [10]. Therefore, Theorem 3.1 tells class $C_{T H}$ of positive threshold functions satisfies

$$
\Lambda_{T H}(n)=1 .
$$

Next, we generalize the concept of threshold functions by introducing some margin in the threshold value. A positive function $f$ is called a $\Delta$-partial threshold function [10] if $f$ is represented by

$$
f(x)= \begin{cases}1, & \text { if } \sum w_{i} x_{i} \geq t+\alpha \\ 0, & \text { if } \sum w_{i} x_{i}<t-\alpha \\ 0 \text { or } 1, & \text { otherwise, }\end{cases}
$$

where $w_{i}(i=1,2, \ldots, n), t, \Delta$ are nonnegative real numbers, and

$$
\alpha=\Delta \min _{i} w_{i}
$$

In this definition, the value $f(x)$ in the case of "otherwise" can be arbitrary, provided that the resulting $f$ is positive. Let

$C_{\triangle P T H}: \quad$ class of $\Delta$-partial positive threshold functions.

For this class, we have the next result.

Theorem 3.2 [9] Class $C_{\triangle P T H}$ satisfies

$$
\Lambda_{\triangle P T H}(n) \leq\lceil\Delta\rceil+1 .
$$

\subsection{Matroid functions}

For a given vector $v \in\{0,1\}^{n}$, we use notations $O N(v)=\left\{j \mid v_{j}=1\right\}$ and $O F F(v)=$ $\left\{j \mid v_{j}=0\right\}$. A positive function $f$ is called a matroid function if for each $v, w \in \min T(f)$ and each $i \in O N(v) \backslash O N(w)$, there exists a $j \in$ $O N(w) \backslash O N(v)$ such that $v-e_{i}+e_{j} \in \min T(f)$. In other words, $M=(E, \mathcal{F})$ forms a matroid [13], where $E=\{1,2, \ldots, n\}$ and $\mathcal{F}=\{O N(v) \mid v \leq$ $a$ for some $a \in \min T(f)\}$. Let

$C_{M A T}:$ the class of matroid functions.

Theorem 3.3 [9] Class $C_{M A T}$ of matroid functions satisfies

$$
\Lambda_{M A T}(n)= \begin{cases}1, & n=1,2,3 \\ 2, & n \geq 4 .\end{cases}
$$

\section{3 $k$-tight positive functions}

A positive function $f$ is called $k$-tight if it satisfies $\max \{\|a-b\| \mid a \in \min T(f), b \in \max F(f)$ and $a-e_{j} \leq b$ for some $\left.j \in O N(a)\right\} \leq k$,

where $k$ is a positive integer. Let

$C_{k T I}$ : class of $k$-tight positive functions.

For example, a positive threshold function with $w_{\max } \leq k w_{\min }$ is always $k$-tight, where $w_{\max }=$ $\max _{i} w_{i}$ and $w_{\min }=\min _{i} w_{i}$, since for any $a \in$ $\min T(f), j \in O N(a)$ and $i_{l} \in O F F(a)(l=$ $1,2, \ldots, k)$,

$$
\begin{aligned}
& \sum_{i=1}^{n} w_{i} a_{i}-w_{j}+\sum_{l=1}^{k} w_{i_{l}} \\
& \quad \geq \sum w_{i} a_{i}-w_{\max }+k w_{\min } \geq \sum w_{i} a_{i} \geq t
\end{aligned}
$$

i.e., $a-e_{j}+\sum_{l=1}^{k} e_{i_{l}} \in T(f)$. To introduce other types of $k$-tight functions, define the rank of a set $S \subseteq\{0,1\}^{n}$ by $r(S)=\max \{\|x\| \mid x \in S\}$ and the anti-rank by $\operatorname{ar}(S)=\min \{\|x\| \mid x \in S\}$, respectively. Then a positive function $f$ satisfying one of the following conditions is $k$-tight.

(i) $|r(\max F(f))-\operatorname{ar}(\min T(f))| \leq k-2$.

(ii) $\operatorname{ar}(\min T(f)) \geq n-k+1$.

(iii) $r(\max F(f)) \leq k-1$.

These types of functions are discussed in [5] and other papers.

Theorem 3.4 [9] Class $C_{k T I}$ of $k$-tight positive functions satisfies

$$
\Lambda_{k T I}(n) \leq k .
$$

\section{General positive functions and positive $k$-DNF func- tions .}

Here we consider the class $C_{P}$ of all positive functions, and

$$
C_{k D N F}: \quad \text { class of positive } k \text {-DNF functions, }
$$

where a positive function $f$ is a positive $k$-DNF function if $\|v\| \leq k$ for all $v \in \min T(f)$. It turns out that these classes do not have constant maximum latency. 


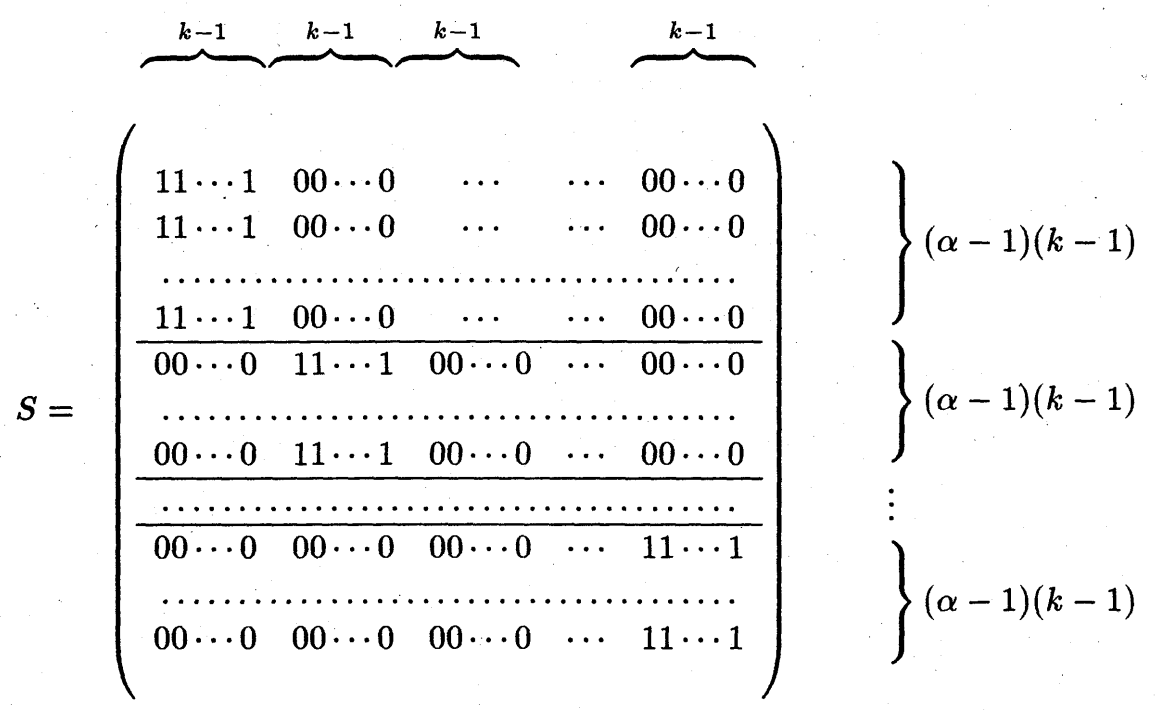

As noted before, this tells that the existence of an incrementally polynomial identification algorithm cannot be concluded from our approach. However, it dose not imply the nonexistence of such algorithm, and in fact it is known [5] that class $C_{k D N F}$ has an incrementally polynomial identification algorithm, which is based on different idea.

Theorem 4.1 $[8,9]$ Class $C_{P}$ of general positive functions satisfies

$$
\lfloor n / 4\rfloor+1 \leq \Lambda_{P}(n) \leq\lceil n / 2\rceil .
$$

For our purpose, the lower bound is more interesting, and it was shown in [9] by construction. We omit its description because the proof of Theorem 4.2 below also contains a similar construction. Also we conjecture $\Lambda_{P}(n)=\lfloor n / 4\rfloor+1$, since $\Lambda_{P}(n) \leq\lfloor n / 4\rfloor+1$ can be shown if we add a rather weak assumption on the set of unknown vectors $U(g)[8]$.

Theorem 4.2 Class $C_{k D N F}$ satisfies, for $n \geq$ $4(k-1)$,

$$
\Lambda_{k D N F}(n) \geq(k-1)\left\lfloor\sqrt{\frac{n}{(k-1)}}\right\rfloor-k+2 .
$$

Proof. For $k=1$, it is clear that $\Lambda_{1 D N F}(n) \geq$ 1 . For $k \geq 2$, we provide an example of $g$ with $\lambda(g)=(k-1)\left\lfloor\sqrt{\frac{n}{(k-1)}}\right\rfloor-k+2$ for $n \geq 4(k-1)$. Let $\alpha=\left\lfloor\sqrt{\frac{n}{(k-1)}}\right\rfloor$, where $\alpha$ satisfies $\alpha \geq 2$. Then

$$
\sqrt{\frac{n}{(k-1)}} \geq \alpha, \text { i.e., } n \geq \alpha^{2}(k-1) .
$$

Therefore, let $n=\alpha^{2}(k-1)+\beta$, where $\beta$ is a nonnegative integer. Now define a $\alpha(\alpha-1)(k-$ 1) $\times n$ matrix:

$$
X=\left(\begin{array}{l|l|l}
S \mid I_{\alpha(\alpha-1)(k-1)} & O_{\alpha(\alpha-1)(k-1) \times \beta}
\end{array}\right),
$$

where $S$ is the $\alpha(\alpha-1)(k-1) \times \alpha(k-1)$ matrix as above. Here, $I_{j}$ is the $j \times j$ unit matrix, and $O_{i \times j}$ is the $i \times j$ zero matrix. Define $f$ by

$$
\min T(f)=(\text { the set of rows of matrix } X),
$$

and a partial function $g$ of $f$ by $M T=\min T(f)$ and $M F=\max F(f) \backslash\{u\}$, where

$$
u=(\underbrace{1,1, \cdots, 1}_{\alpha(k-1)}, \underbrace{0,0, \cdots, 0}_{\alpha(\alpha-1)(k-1)}, \underbrace{1,1, \cdots, 1}_{\beta}) .
$$

Then $u+e_{j} \in T(M T)$ for any $j \in O F F(u)$ since $u \in \max F(f)$ and $M T=\min T(f)$. Moreover, $u-e_{j} \in F(M F)$ For any $j \in\{1,2, \ldots, \alpha(k-1)\}$, and $u-\sum_{j \in S} e_{j} \notin F(M F)$, where $S=\{n-\beta+$ $1, n-\beta+2, \ldots, n\}$. In other words,

$$
U(g)=\{(\underbrace{1,1, \cdots, 1}_{\alpha(k-1)}, \underbrace{0,0, \cdots, 0}_{\alpha(\alpha-1)(k-1)}, \underbrace{*, *, \cdots, *}_{\beta})\},
$$

where $*$ stands for 0 or 1 . It is not difficult to see that $\|a-w\|=(\alpha-1)(k-1)+1$ for every $a \in M T$, and $w \in U(g)$ and $\|b-w\|=(\alpha-$ 
$1)(k-1)+1$ for every $b \in M F$ and $w \in U(g)$. Therefore, its latency is

$$
\begin{aligned}
\lambda(g) & =(\alpha-1)(k-1)+1 \\
& =(k-1)\left\lfloor\sqrt{\frac{n}{(k-1)}}\right\rfloor-k+2 .
\end{aligned}
$$

\section{Discussion}

In this paper, we introduced the maximum latency as a measure for the difficulty to find an unknown vector. Several interesting subclasses of positive functions have constant maximum latency. It would be important to find other subclasses of positive functions with constant maximum latency. Of course, the ultimate goal is to develop a polynomial time identification algorithm for general positive functions (or to disprove its existence) by some new tools.

\section{Acknowledgments}

The authors are grateful to the valuable comments given by Associate Professor H. Nagamochi and the members of our laboratory. This research was partially supported by the Scientific Grant-in-Aid from Ministry of Education, Science and Culture of Japan.

\section{References}

[1] D. Angluin, Queries and concept learning, Machine Learning, 2 (1988) 319-342.

[2] J. C. Bioch and T. Ibaraki, Complexity of identification and dualization of positive Boolean functions, RUTCOR Research Report RRR 25-93, Rutgers University, 1993.

[3] E. Boros, P. L. Hammer, T. Ibaraki and K. Kawakami, Identifying 2-monotonic positive Boolean functions in polynomial time, ISA'91 Algorithms, edited by W. L. Hsu and R. C. T. Lee, Springer Lecture Notes in Computer Science, 557 (1991) 104-115.
[4] Y. Crama, P. L. Hammer and T. Ibaraki, Cause-effect relationships and partially defined boolean functions, Annals of Operations Research, 16 (1988) 299-326.

[5] T. Eiter and G. Gottlob, Identifying the minimal transversals of a hypergraph and related problems, Technical Report CDTR 91/16, Christial Doppler Labor für Expertensysteme, Technische Universität Wien, January 1991.

[6] T. Ibaraki and T. Kameda, A Theory of coteries: Mutual exclusion in distributed systems, IEEE Trans. on Parallel and Distributed Systems, 4 (1993) 779-794.

[7] D. S. Johnson, M. Yannakakis and C. H. Papadimitriou, On generating all maximal independent sets, Information Processing Letters, 27 (1988) 119-123.

[8] K. Makino and T. Ibaraki, The maximum latency of partially defined positive Boolean functions, Trans. IEICE, J76-D-1 (1993) 409-416 (in Japanese).

[9] K. Makino and T. Ibaraki, The maximum latency and identification of positive Boolean functions, Technical Report, Kyoto University, in preparation.

[10] S. Muroga, Threshold Logic and Its Applications, Wiley-Interscience, 1971.

[11] R. Reiter, A theory of Diagnosis from first principles, Artificial Intelligence, 32 (1987) 57-95.

[12] L. G. Valiant, A theory of the learnable, Communications of the ACM, 27 (1984) 1134-1142.

[13] D. J. A. Welsh, Matroid Theory, Academic Press, 1976. 